\title{
MLC1 is associated with the Dystrophin-Glycoprotein Complex at astrocytic endfeet
}

\author{
Ilja Boor · Machiel Nagtegaal · Wouter Kamphorst $\cdot$ Paul van der Valk $\cdot$ Jan C. Pronk • \\ Jack van Horssen • Argirios Dinopoulos • Kevin E. Bove • Ignacio Pascual-Castroviejo • \\ Francesco Muntoni $\cdot$ Raúl Estévez $\cdot$ Gert C. Scheper · Marjo S. van der Knaap
}

Received: 8 January 2007 / Revised: 16 May 2007 / Accepted: 1 June 2007 / Published online: 13 July 2007

(C) Springer-Verlag 2007

\begin{abstract}
Megalencephalic leukoencephalopathy with subcortical cysts (MLC) is a progressive cerebral white matter disease with onset in childhood, caused by mutations in the $M L C 1$ gene. MLC1 is a protein with unknown function that is mainly expressed in the brain in astrocytic endfeet at the blood-brain and cerebrospinal fluid-brain barriers. It shares its localization at astrocytic endfeet with the dystrophin-associated glycoprotein complex (DGC). The objective of the present study was to investigate the possible association of MLC1 with the DGC. To test this
\end{abstract}

Financial support was received from the Dutch Organization for Scientific Research (ZonMw, grant 903-42-097), The Hersenstichting (grants 10F02(2).02 and 13F05.04), the Optimix Foundation for Scientific Research, the Fundació La Caixa and FIS PI04/1680. RE is a Ramón y Cajal researcher. The Muscular dystrophy campaign grant to FM is also gratefully acknowledged.

I. Boor · M. Nagtegaal · G. C. Scheper · M. S. van der Knaap ( $₫)$

Department of Pediatrics/Child Neurology,

VU University Medical Center, De Boelelaan 1117,

1081 HV Amsterdam, The Netherlands

e-mail:ms.vanderknaap@vumc.nl

W. Kamphorst · P. van der Valk

Department of Pathology, VU University Medical Center,

Amsterdam, The Netherlands

J. C. Pronk

Department of Human Genetics,

VU University Medical Center,

Amsterdam, The Netherlands

J. van Horssen

Department of Molecular Cell Biology,

VU University Medical Center,

Amsterdam, The Netherlands

\section{A. Dinopoulos}

Department of Pediatric Neurology,

VU University Medical Center,

Amsterdam, The Netherlands hypothesis, (co)-localization of DGC-proteins and MLC1 was analyzed by immunohistochemical stainings in gliotic brain tissue from a patient with multiple sclerosis, in glioblastoma tissue and in brain tissue from an MLC patient. In control tissue, a direct protein interaction was tested by immunoprecipitation. Results revealed that MLC1 is co-localized with DGC-proteins in gliotic brain tissue. We demonstrated that both MLC1 and aquaporin-4, a member of the DGC, were redistributed in glioblastoma cells. In MLC brain tissue, we showed absence of MLC1 and altered expression of several DGC-proteins. We demonstrated a direct protein interaction between MLC1 and Kir4.1. From these results we conclude that MLC1 is associated with the DGC at astrocytic endfeet.

Keywords MLC1 - Astrocytes - Endfeet · Dystrophinassociated glycoprotein complex $\cdot$ Leukodystrophy

\author{
K. E. Bove \\ Department of Pathology, \\ Cincinnati Children's Hospital Medical Center, \\ Cincinnati, USA \\ I. Pascual-Castroviejo \\ Department of Pediatric Neurology, \\ Children's Hospital La Paz, Madrid, Spain \\ F. Muntoni \\ The Dubowitz Neuromuscular Centre, \\ Hammersmith Hospital, \\ Imperial College, London, UK \\ R. Estévez \\ Department of Biochemistry and Molecular Biology, \\ Faculty of Biology and Barcelona Science Park, \\ Barcelona, Spain
}




\section{Introduction}

Megalencephalic leukoencephalopathy with subcortical cysts (MLC, MIM 604004) is a childhood leukoencephalopathy with autosomal recessive inheritance [21]. Patients develop macrocephaly during the first year of life, followed by slow motor deterioration with cerebellar ataxia and spasticity [21]. Magnetic resonance imaging (MRI) shows diffusely abnormal, mildly swollen cerebral white matter and subcortical cysts in anterior temporal and frontoparietal areas [21]. Histopathologic and electron microscopic examination of brain tissue reveals countless intramyelinic vacuoles [22].

From early on, the striking similarity in MRI features of MLC and congenital muscular dystrophy (CMD) with merosin deficiency (CMD type 1A, MDC1A) was noted [21]. Patients with MDC1A also have diffusely abnormal, mildly swollen cerebral white matter and sometimes anterior temporal cysts (Fig. 1) [23]. Microscopic examination of the brain of a patient, who was later genetically confirmed to have MDC1A (B. Echenne, personal communication) also revealed myelin vacuolation [8]. MDC1A is caused by mutations in the LAMA2 gene, encoding the laminin- $\alpha 2$ chain of merosin [9]. In contrast to MDC1A, MLC is not associated with muscle disease [21]. We hypothesized that MLC could be the "brain-only" variant of MDC1A. We could, however, not confirm linkage of MLC to the LAMA2 locus and instead demonstrated that mutations in another gene, $M L C 1$, cause MLC [10]. $M L C 1$ encodes a plasmamembrane protein of unknown function. Recently, the MLC1-protein was found to be located in astrocytic endfeet at the blood-brain and cerebrospinal fluid-brain barriers [3, 19].

Other proteins localized at astrocytic endfeet are those of a multi-subunit complex called dystrophin-associated glycoprotein complex (DGC) [12, 20]. The DGC is expressed in various tissues and has been characterized best in skeletal muscle where it connects the cytoskeleton of muscle fibers to the extracellular matrix [14]. Mutations in different DGC components lead to various muscular dystrophies. In a subset of muscular dystrophies, the congenital muscular dystrophies (CMDs), the muscular phenotype is often combined with brain involvement, including white matter abnormalities, most notably in MDC1A [23].

The brain DGC is involved in the adhesion of perivascular glia to the extracellular matrix [20]. At the astrocytic endfeet, the DGC consists of four main components $[6,16$, 29]: (1) $\alpha$ - and $\beta$-dystroglycan, which form the backbone of the DGC by forming a transmembrane link between the actin cytoskeleton and the extracellular matrix. $\alpha$-Dystroglycan is a heavily glycosylated peripheral membrane protein that binds to extracellular ligands such as merosin
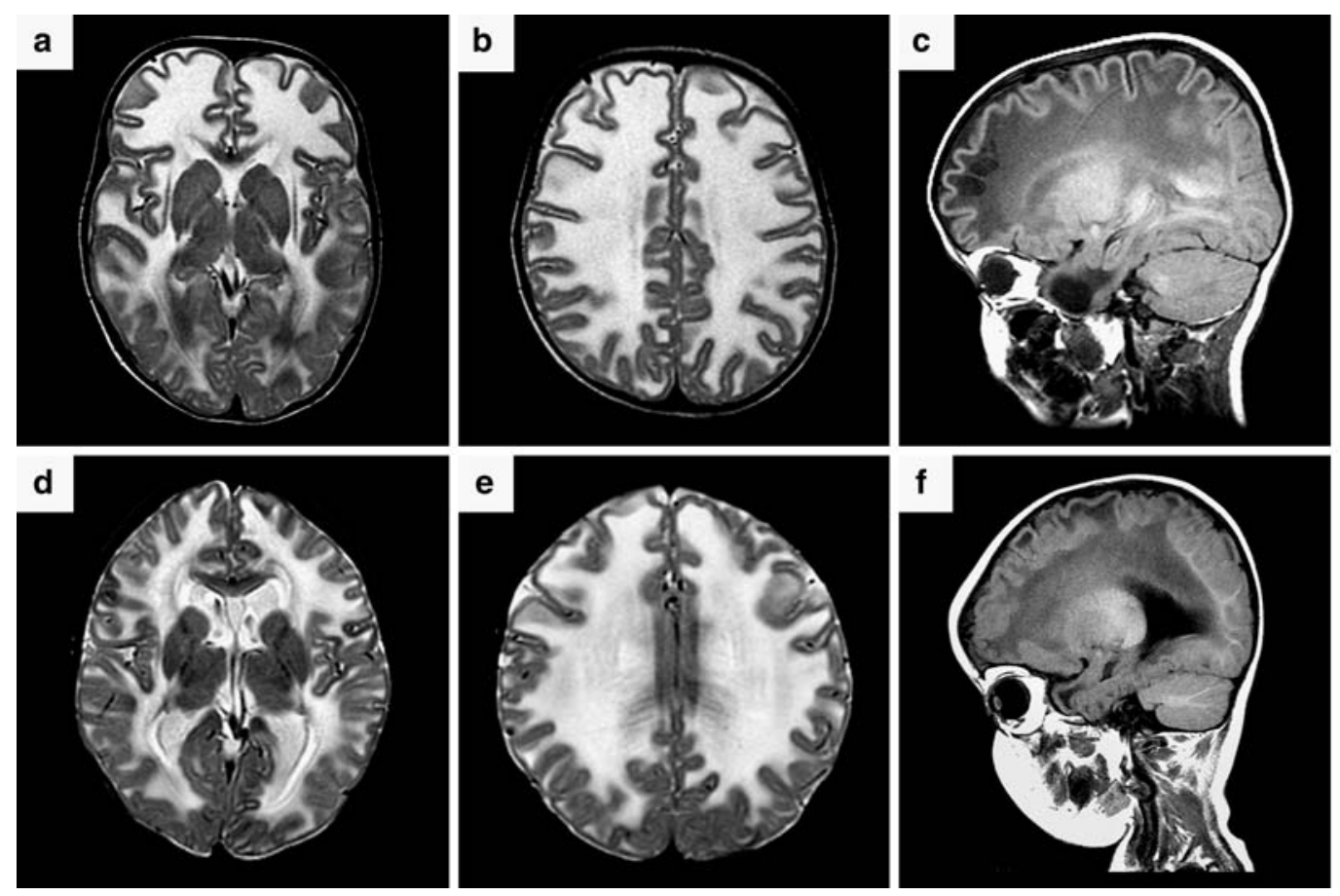

MLC

MDC1A

Fig. 1 MRI of the brain of a patient with MLC (a-c) and MDC1A (d-f). The axial T2-weighted images $(\mathbf{a}, \mathbf{b}, \mathbf{d}, \mathbf{e})$ reveal extensive signal abnormalities and mild swelling of the cerebral white matter in both $\operatorname{MLC}(\mathbf{a}, \mathbf{b})$ and $\operatorname{MDC} 1 \mathrm{~A}(\mathbf{d}, \mathbf{e})$. The corpus callosum and internal capsule are relatively preserved in both cases $(\mathbf{a}, \mathbf{d})$. The sagittal
T1-weighted images reveal a large cyst in the anterior temporal white matter and smaller cysts in the frontal subcortical white matter in the MLC patient (c). There are small cysts in the same regions in the MDC1A patient (f). Both MLC and MDC1A patients had a genetically confirmed diagnosis 
and agrin. $\beta$-Dystroglycan is a transmembrane protein that anchors the extracellular $\alpha$-dystroglycan to the plasma membrane and interacts intracellularly with dystrophin (and/or utrophin, which can replace dystrophin) and syntrophin. (2) Dystrophin and/or utrophin, are linker molecules that can form a bridge between actin and $\beta$-dystroglycan. (3) $\alpha$-Dystrobrevin, which is linked to dystrophin and/or utrophin binds to syntrophin. (4) Syntrophin, which binds to the water channel aquaporin- 4 and the potassium channel Kir4.1 is responsible for the polarized expression of these proteins in astrocytic endfeet.

The striking similarities in brain MRI features and pathology between MLC and MDC1A, together with the specific localization of both MLC1 and merosin at astrocytic endfeet, brought us to the hypothesis that there may be an association between MLC1 and DGC in the brain. In the present paper we report on our studies to test this hypothesis. We investigated the co-localization of MLC1 and DGC-proteins in gliotic white matter, because MLC1 staining is more intense in gliotic brain tissue than in normal tissue [3]. Previous studies in humans and mice have shown that if members of the DGC are mutated or missing, the complex may be disrupted, which affects the localization of other protein members [1, 13, 16, 27]. We therefore also tested our hypothesis by studying the localization of MLC1 and several DGC-proteins in glioblastoma and MLC brain tissue. Besides, we assessed a direct association between MLC1 and DGC-members by co-immunoprecipitation experiments.

\section{Materials and methods}

Immunohistochemical staining of human brain tissue

Human control brain tissue specimens, both frozen and paraffin-embedded, consisting of neocortex and white matter, were obtained at autopsy from patients without neurological disease and neuropathologic abnormalities. Additionally, frozen brain tissue was obtained from a multiple sclerosis patient to study gliotic white matter in which MLC1 has a normal localization but MLC1 expression is increased [3]. During life, all patients or their next of kin had given consent for autopsy and the use of brain tissue for research purposes.

Human glioblastoma multiforme tissue (frozen) and brain biopsy tissue from MLC patients (both frozen and paraffin-embedded) were obtained for diagnostic purposes and used for research purposes with informed consent of patients and families. Light-microscopy of glioblastoma tissue was used to select areas that display characteristic glioblastoma features, including necrosis, giant cells and endothelial proliferation, for further studies. Brain biopsies were obtained in the following MLC patients: EL18 (decreased MLC1 expression [2], frozen tissue), EL649 (homozygous for the c.733G > C (p.Ala245Pro) mutation; paraffin-embedded tissue), and EL746 [compound-heterozygous for the c.268-1G $>$ A and c.597 + $1 \mathrm{G}>\mathrm{A}$ mutations, leading to splice-defects and truncation of the protein (p.Cys90-Val107del and p.Val200-Ala238del); paraffinembedded tissue].

Immunohistochemistry was performed as described previously [7] with antibodies against the following proteins: MLC1 (polyclonal; 1:200 [3]); merosin (monoclonal; 1:500 [11]); aquaporin-4 (polyclonal; 1:200, Chemicon, Huissen, The Netherlands); Kir4.1 (polyclonal; 1:200, USBiological, Swampscott, Massachusetts), $\beta$-dystroglycan (monoclonal; 1:100, Santa Cruz, Heidelberg, Germany), $\alpha$-dystroglycan (monoclonal; 1:100, UpstateCellSignaling solutions), agrin (monoclonal; 1:2500 [26]); glial fibrillary acidic protein (GFAP, polyclonal; 1:500, DAKO, Heverlee, Belgium) and NeuN (monoclonal; 1:1600, MAB377, Chemicon). In all slides, except the slides used for double stainings, the nuclei are counterstained with Haematoxylin. Double immunofluorescence staining was performed as described [3] with agrin, $\beta$-dystroglycan (monoclonal; 1:100) or syntrophin (monoclonal; 1:100, Affinity BioReagents, Raamdonksveer, The Netherlands) versus MLC1. In negative controls the primary antibodies were omitted. Doublestainings were performed as described previously [25]. In frozen tissue double-stainings were done with agrin (red) antibodies versus AB4 (blue, polyclonal; 1:200, Lab Vision-NeoMarkers, Fremont, CA, USA) antibodies and in paraffin tissue with agrin or $\alpha$-dystroglycan antibodies versus NeuN or GFAP. For the agrin (brown)/NeuN (blue) double-stainings, agrin was visualized with biotinylated secondary antibodies (DAKO) and NeuN with poly-HRP (Immunologic, Duiven, The Netherlands). For the agrin (red)/GFAP (blue) double-stainings, agrin was detected with poly-HRP (Immunologic) and GFAP with biotinylated secondary antibodies (DAKO). In negative controls the primary antibodies were omitted.

Immuno-precipitation and western blotting

Preparation of brain tissue and immunoprecipitation experiments were performed as previously described [5] without antibodies as a negative control, with $1-15 \mu \mathrm{g}$ mMLC1-C (home made), with the nonspecific CHOP (Santa Cruz, Heidelberg, Germany) as an extra negative control, and with Kir4.1 (USBiological, Swampscott, Massachusetts) antibodies. Western blots were incubated with the Kir4.1 antibodies overnight at $4^{\circ} \mathrm{C}$ and complexes were visualized using ECL Western Blot detection reagents (Amersham, Buckinghamshire, United Kingdom). 


\section{Results}

Co-localization of MLC1 and DGC-members

To test whether MLC1 co-localizes with DGC-proteins at astrocytic endfeet, we performed double-immunofluorescent staining experiments in gliotic brain tissue with the transmembrane protein MLC1 (Fig. 2a, d, g; green) versus the extracellular matrix protein agrin (Fig. 2b; red), the intracellular protein syntrophin (Fig. 2e; red), and the transmembrane protein $\beta$-dystroglycan (Fig. $2 \mathrm{~h}$; red). There was partial overlap (yellow) between all three DGC-proteins (red) and MLC1 (green). The co-localization (Fig. 2i; yellow) of MLC1 (Fig. $2 \mathrm{~g}$; green) and $\beta$-dystroglycan (Fig. 2h; red) was most striking. If MLC1, agrin, $\beta$-dystroglycan or syntrophin was omitted, fluorescent labeling was only visible for the appropriate isotype, thereby excluding cross-reactivity (not shown). On omission of the primary antibody no staining was observed (not shown).

MLC1 and aquaporin-4 are redistributed in glioblastoma cells

Staining normal human brain tissue with anti-MLC1 and anti-aquaporin-4 antibodies confirmed their specific expres- sion around blood vessels, consistent with the previously described perivascular localization (Fig. 3a, c) [3, 15]. In glioblastoma tissue, both MLC1 and aquaporin-4 had altered expression patterns. The proteins were no longer confined to astrocytic endfeet, but were redistributed over the entire glioblastoma cell (Fig. 3b, d). Anti-agrin staining showed expression of the protein in the extracellular matrix around blood vessels of all sizes in normal brain tissue (Fig. 3e), whereas it was exclusively expressed around larger blood vessels in glioblastoma tissue (Fig. 3f). Double staining of agrin (red) with AB4 (blue), an endothelial marker, in glioblastoma tissue confirmed agrin and AB4 staining around larger blood vessels (Fig. 3g) and absence of agrin around small vessels (Fig. $3 \mathrm{~g}$, boxed area). This is in line with previously published results [27]. On omission of the primary antibody no staining was observed (not shown).

Absence of MLC1 and altered expression of DGC-proteins in MLC brain tissue

Anti-MLC1 staining revealed perivascular expression of the protein in control brain tissue (Fig. 4a) [3] and absence of staining in MLC tissue (Fig. 4b). The distribution of merosin, $\beta$-dystroglycan and aquaporin-4 was similar in
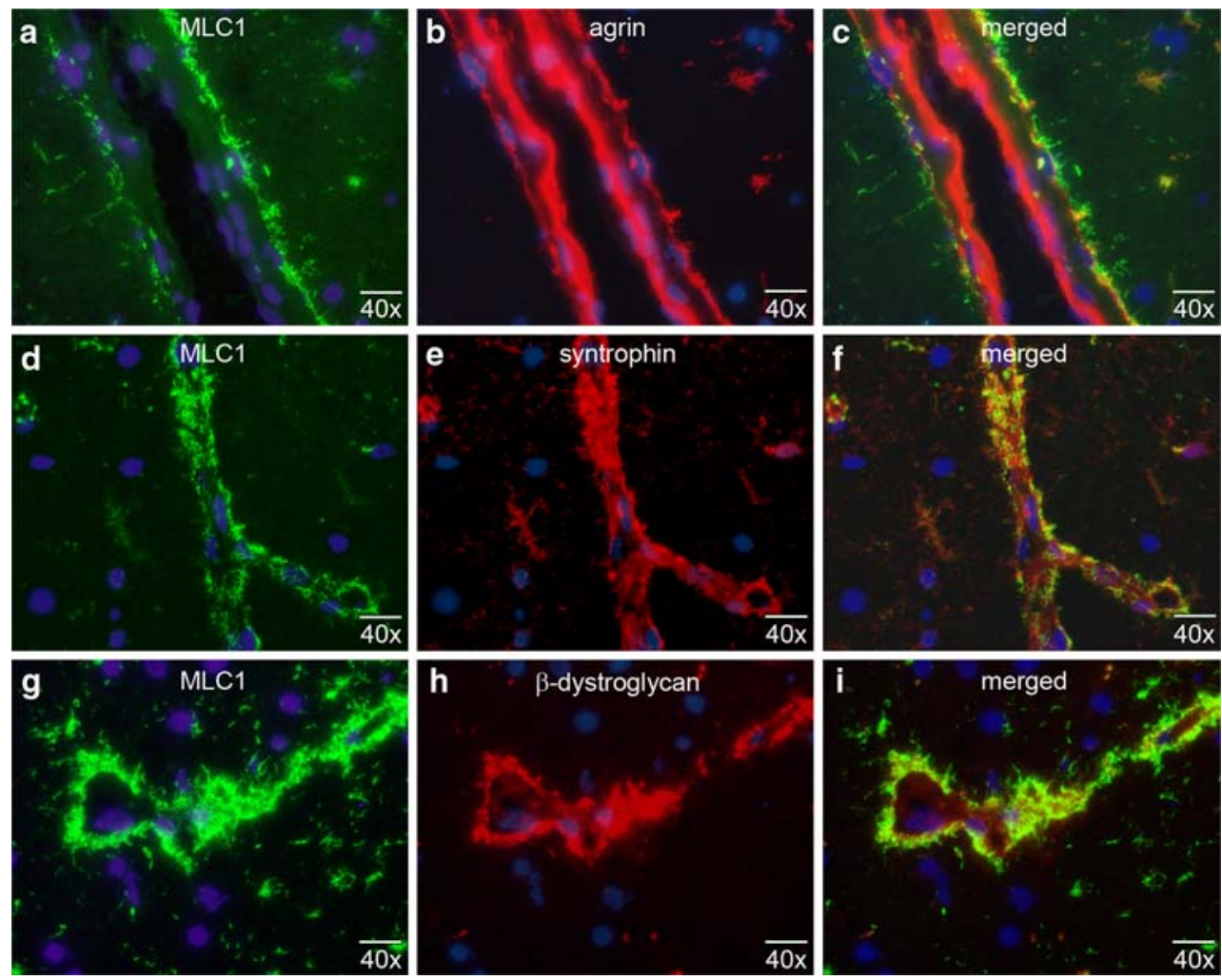

Fig. 2 Co-localization of MLC1 and DGC-proteins. a-i Double immuno-fluorescence labelling of $\operatorname{MLC1}(\mathbf{a}, \mathbf{d}, \mathbf{g}$, green), agrin (b, red), syntrophin (e,red), and $\beta$-dystroglycan $(\mathbf{h}, r e d)$ in gliotic human brain tissue. There is partial overlap (c, f, $\mathbf{i}$, yellow) between
MLC1 and these DGC-proteins. The overlap (i, yellow) between the transmembrane proteins MLC1 (green) and $\beta$-dystroglycan (red) is most striking. In all panels the ocular magnification and $10 \mu \mathrm{m}$ scale bars are indicated in the lower right hand corner 


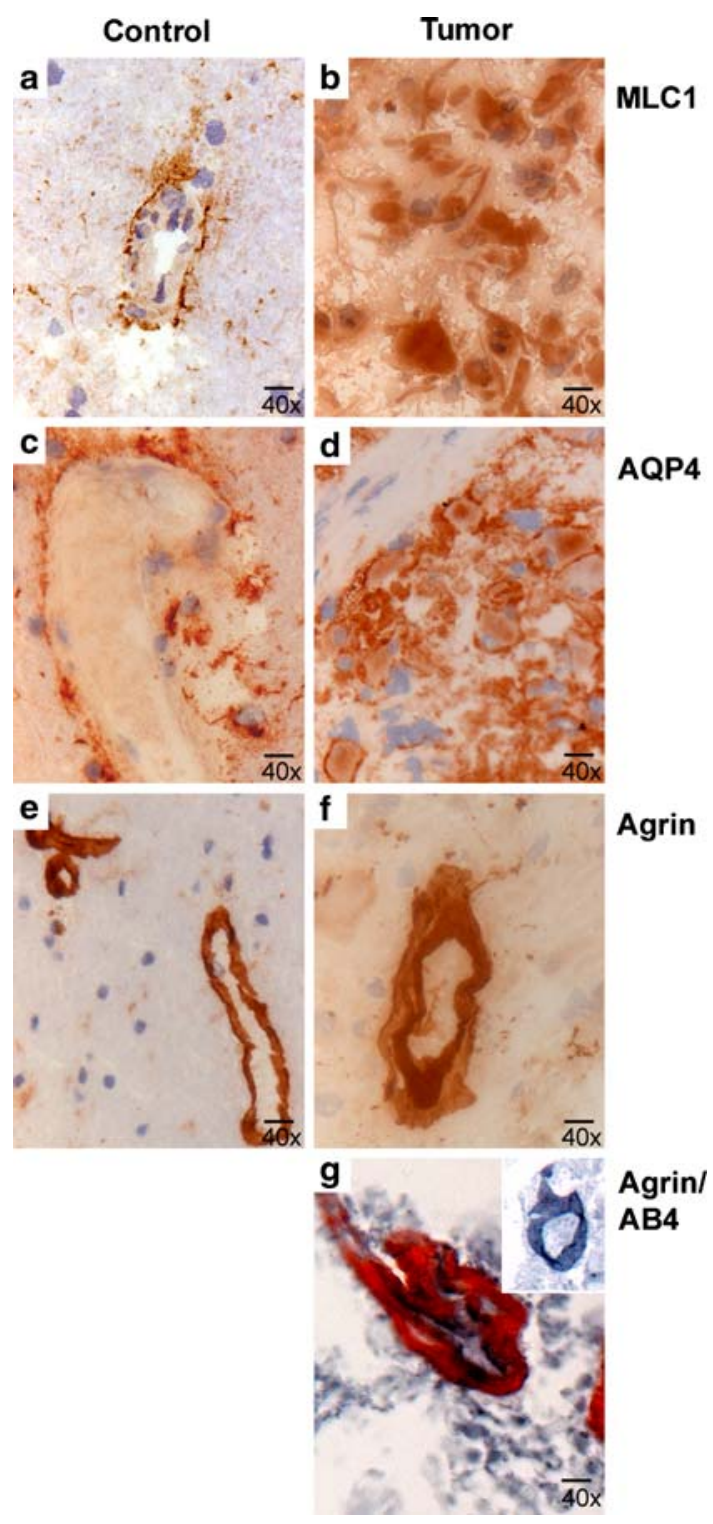

Fig. 3 Localization of MLC1 in glioblastoma tissue. Immunohistochemical staining of MLC1, aquaporin-4 (AQP4) and agrin in control brain (a, c, e) and tumor tissue (b, d, f, g). a MLC1 has a perivascular localization in normal tissue. $\mathbf{b}$ In glioblastoma tissue, the restriction of $M L C 1$ to perivascular areas is lost and the protein has an intracellular location in glioblastoma cells. c $A Q P 4$ is expressed in perivascular astroglial endfeet in control brain. d AQP4 is localized in glioblastoma cells. e, f Agrin is expressed in the extracellular matrix around blood vessels of all sizes in normal tissue (e) and only around large vessels in glioblastoma tissue (f). $\mathbf{g}$ Double-staining of agrin (red)/AB4 (blue) in glioblastoma tissue shows agrin and $A B 4$ (endothelial marker) staining around larger blood vessels. The boxed area in panel $\mathbf{g}$ shows that agrin staining is absent around small vessels. In all panels the ocular magnification and $10 \mu \mathrm{m}$ scale bars are indicated in the lower right corner

control (Fig. 4c, e, g) and MLC tissue (Fig. 4d, f, h). In control tissue, Kir4.1 showed a perivascular localization in configuration suggesting presence in distal atroglial processes (Fig. 4i), while in MLC patient tissue Kir4.1 additionally showed a diffuse cytoplasmic staining of astrocytes (Fig. 4j). In control tissue, agrin (Fig. 4k) and $\alpha$-dystroglycan (Fig. $4 \mathrm{~m}$ ) were seen in the perivascular extracellular matrix, whereas these proteins were observed both in the perivascular extracellular matrix and within cells in MLC tissue (Fig. 4l, n).

The cells expressing these latter two DGC-proteins had the morphological characteristics of neurons. Double stainings of agrin and $\alpha$-dystroglycan with NeuN, a neuronal marker, in MLC brain tissue confirmed that both agrin (brown) (Fig. 4o) and $\alpha$-dystroglycan (not shown) co-localized with NeuN (blue). Although, most redistribution appeared to be in neurons, double stainings of agrin (red) and GFAP (blue) showed that also some astrocytes displayed positive cell body staining for agrin (Fig. 4p). On omission of the primary antibody no staining was observed (not shown).

\section{Direct protein interaction between MLC1 and Kir4.1}

To verify interaction of DGC-proteins with MLC1, immunoprecipitation experiments were performed using human control brain lysates. Figure 5 shows that after immunoprecipitation with both anti-MLC1 and anti-Kir4.1 antibodies and probing the precipitate for the presence of Kir4.1, a band at $200 \mathrm{kDA}$ was detected, the size expected for Kir4.1. This band was not present in a pull-down without antibodies or with an unrelated antibody (anti-CHOP). Of concern in this experiment was the use of cross-linking reagents, since this might cause nonspecific interactions. When cross-linking reagents were left out, we failed to detect interactions, which is in agreement with previous studies on interaction between DGC-members [5].

\section{Discussion}

The DGC is important in linking cytoskeletal proteins to the extracellular basal lamina. It is present in several tissues including muscle, heart, nerve and brain. The composition of this complex shows some differences for different tissues. Structural defects in a number of DGC-members cause muscular dystrophies. Best known is Duchenne muscular dystrophy, related to mutations in dystrophin. Another muscular dystrophy is the CMD MDC1A, in which the MRI features are strikingly similar to those of MLC. MDC1A is caused by mutations in the laminin- $\alpha 2$ chain of merosin [9]. A specific group of CMD variants is known as "dystroglycanopathies" and includes Walker-Warburg syndrome, Fukuyama type of CMD, muscle-eye-brain disease, CMD type $1 \mathrm{C}$ and type $1 \mathrm{D}$. They are characterized by hypoglycosylation of $\alpha$-dystroglycan [14]. MDC1A and most of the dystroglycanopathies lead to both muscle disease and brain involvement, including cerebral white matter abnormalities. 

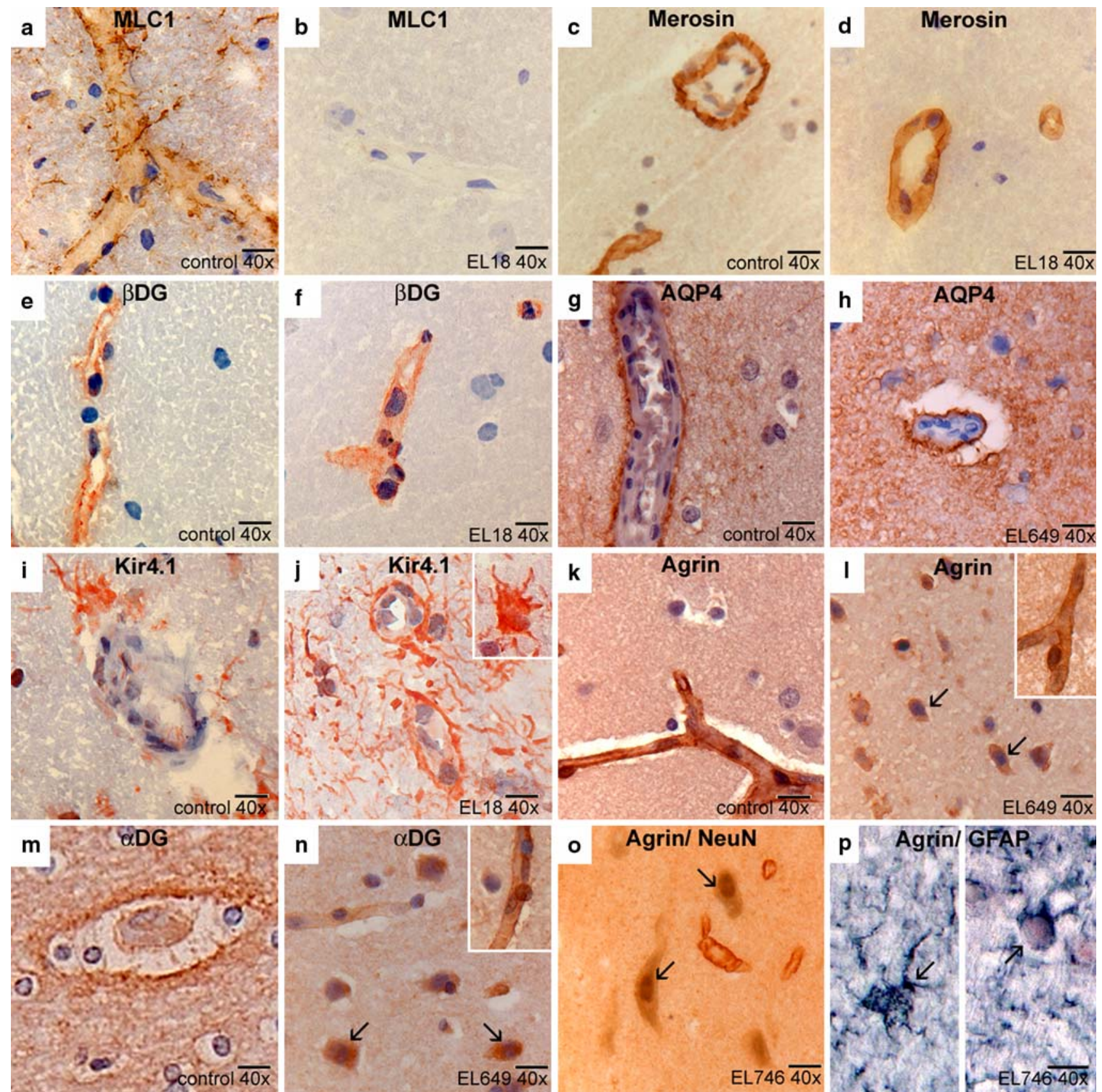

Fig. 4 Localization of proteins associated with the DGC in MLC patients. In control tissue the perivascular staining of $M L C 1$ is visible (a), but no staining is observed in MLC brain tissue (b). Normal perivascular basal lamina staining with merosin is seen in control (c) and MLC (d) brain tissue. $\beta$-dystroglycan has a normal perivascular distribution in both control (e) and MLC (f) brain tissue. Aquaporin-4 (AQP4) has a normal perivascular distribution in both control (g) and MLC (h) brain tissue. Kir4.1 is present in distal astrocytic processes around blood vessels in control tissue (i). In MLC tissue (j) Kir4.1 is expressed both in distal astrocytic processes around blood vessels and in cell bodies of astrocytes (boxed area). Agrin is expressed in the extracellular matrix around blood vessels in control tissue (k). In MLC tissue (l) agrin is expressed in both the extracellular matrix around

blood vessels (boxed area) and in cells, as indicated by arrows. Immunostaining for $\alpha$-dystroglycan shows a normal perivascular distribution in control tissue (m) and expression of $\alpha$-dystroglycan around blood vessels (boxed area) and in cells in MLC tissue (arrows in $\mathbf{n}$ ). o Double-staining of NeuN (blue)/agrin (brown) in MLC tissue shows cytoplasmic agrin staining in NeuN positive cells (arrows). p Double-staining of Agrin (red)/GFAP (blue) in MLC brain tissue. The picture on the left shows an astrocyte that is only GFAP positive (arrow), while the picture on the right shows an astrocyte that is both GFAP and agrin positive (arrow, brown staining in cell body). In all panels the ocular magnification, $10 \mu \mathrm{m}$ scale bars and patient numbers are indicated in the lower right corner 


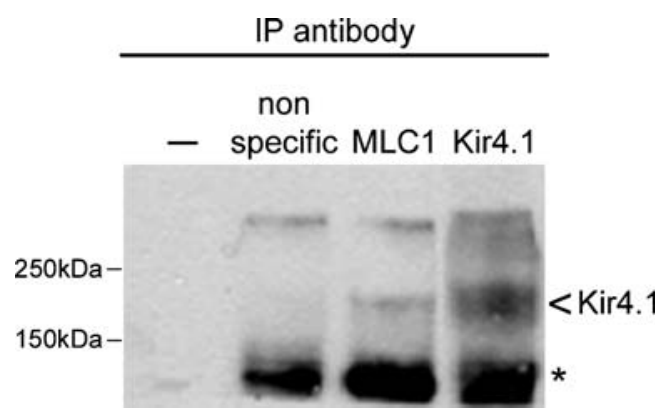

Fig. 5 Co-immunoprecipitation $(I P)$ of human brain lysates. Antibodies used for IP are indicated above the figure. The dash indicates pulldown without antibodies; anti-CHOP was used as a nonspecific antibody. The resulting Western blot was probed with antibodies against Kir4.1. The arrowhead shows the position of Kir4.1 at $200 \mathrm{kDa}$ and the asterisk indicates a nonspecific band that is pulled down with all the antibodies used

We hypothesized that MLC1 could be associated with the DGC in the brain. To strengthen our hypothesis, we showed co-localization between MLC1 and DGC-proteins around blood vessels by immunofluorescent stainings. A common feature of the DGC is that mutations affecting one of its components often lead to destabilization of the complex with reduced or altered expression of other DGCmembers. For instance, in muscle and brain tissue of patients with a dystroglycanopathy, immunostaining is reduced for $\alpha$-dystroglycan as well as merosin and agrin [13]. In patients with Duchenne muscular dystrophy and Fukuyama type of CMD, decreased expression of multiple DGC-proteins has been found in muscle and brain [4, 17, 18]. In brain tissue of syntrophin, knock out mice altered expression of aquaporin-4 has been reported [16]. In glioblastoma tissue, abnormal expression of aquaporin- 4 and syntrophin over the entire surface of glioblastoma cells and absence of $\alpha$-dystroglycan from glial structures have been reported [27, 28]. Agrin was only present around larger vessels but absent around small vessels in glioblastoma tissue $[27,28]$. We decided to use these DGC destabilization phenomena to test our hypothesis. Because of the co-localization of MLC1 and DGC-members at perivascular regions and the disassembly of the DGC in glioblastoma tissue we were interested in whether MLC1 is redistributed in this type of brain tumor. We, therefore, tested the expression pattern of MLC1 by immunohistochemical staining in glioblastoma tissue. We confirmed previous findings for agrin and aquaporin-4 [27, 28] and demonstrated that MLC1, like aquaporin- 4 , is redistributed in glioblastoma cells. We were also interested whether MLC1 mutations would lead to destabilization of the complex with reduced or altered expression of DGC-members in MLC brain. We demonstrated the absence of MLC1 and altered expression of Kir4.1, agrin and $\alpha$-dystroglycan in brain tissue of MLC patients, whereas merosin, $\beta$-dystroglycan and aquaporin-4 retained their normal perivascular localization. Agrin and $\alpha$-dystroglycan were redistributed in both neurons and astrocytes, although most positive staining cells appeared to be neurons. All the above results provide circumstantial evidence for an association between MLC1 and the DGC. To show a direct protein-protein interaction we performed co-immunoprecipitation experiments that revealed an association between MLC1 and Kir4.1. All together, the above findings provide strong evidence for an association between MLC1 and the DGC.

So far almost all defects in DGC-proteins have been associated with a muscular dystrophy, which is absent in MLC. This difference can be ascribed to a difference in DGC composition for muscle as compared to brain. Whereas merosin is expressed both in muscle and brain, MLC1 is not expressed in muscle (http://biomed.ngic.re.kr/ cgi-bin/cards/carddisp?MLC1\&search = KIAA0027) Both in MLC and MDC1A, the water content of the affected white matter is abnormally high due to intramyelinic vacuole formation $[8,22,24]$. The DGC is crucial for anchoring of water and potassium channels at the perivascular endfeet [15]. Destabilization of the DGC may lead to changes in glial polarity, disturbances of the blood-brain barrier and alterations in ion and water homeostasis of the brain [27, 28], and result in an increased myelin water content. Our findings may, therefore, have implications for the pathophysiology of white matter abnormalities observed in MLC, MDC1A and the dystroglycanopathies.

Acknowledgments We thank Prof. Dr. Annemieke Rozemuller for providing the glioblastoma tissue and the Netherlands Brain Bank, Amsterdam, for supplying control human brain tissue. We thank the following people for their gracious gift of antibodies: Jaap van den Born (anti-agrin), Ilmo Leivo and Thea Tadema (anti-NeuN). We are grateful to Dr. Brenda Wong for providing us with the MRI of a patient with merosin-negative congenital muscular dystrophy and anterior temporal cysts and Dr. Bernard Echenne for his personal communication on the genetic confirmation of this patient.

\section{References}

1. Bonuccelli G, Sotgia F, Schubert W, Park DS, Frank PG, Woodman SE, Insabato L, Cammer M, Minetti C, Lisanti MP (2003) Proteasome inhibitor (MG-132) treatment of mdx mice rescues the expression and membrane localization of dystrophin and dystrophin-associated proteins. Am J Pathol 163:1663-1675

2. Boor PK, de Groot K, Mejaski-Bosnjak V, Brenner C, van der Knaap MS, Scheper GC, Pronk JC (2006) Megalencephalic leukoencephalopathy with subcortical cysts: an update and extended mutation analysis of MLC1. Hum Mutat 27:505-512

3. Boor PK, de Groot K, Waisfisz Q, Kamphorst W, Oudejans CB, Powers JM, Pronk JC, Scheper GC, van der Knaap MS (2005) MLC1: a novel protein in distal astroglial processes. J Neuropathol Exp Neurol 64:412-419

4. Brockington M, Blake DJ, Prandini P, Brown SC, Torelli S, Benson MA, Ponting CP, Estournet B, Romero NB, Mercuri E, Voit T, Sewry CA, Guicheney P, Muntoni F (2001) Mutations in 
the fukutin-related protein gene (FKRP) cause a form of congenital muscular dystrophy with secondary laminin alpha2 deficiency and abnormal glycosylation of alpha-dystroglycan. Am J Hum Genet 69:1198-1209

5. Connors NC, Adams ME, Froehner SC, Kofuji P (2004) The potassium channel Kir4.1 associates with the dystrophin-glycoprotein complex via alpha-syntrophin in glia. J Biol Chem 279:28387-28392

6. Culligan K, Ohlendieck K (2002) Diversity of the brain dystrophin-glycoprotein complex. J Biomed Biotechnol 2:31-36

7. De Groot CJ, Montagne L, Janssen I, Ravid R, Van Der Valk P, Veerhuis $R$ (2000) Isolation and characterization of adult microglial cells and oligodendrocytes derived from postmortem human brain tissue. Brain Res Brain Res Protoc 5:85-94

8. Echenne B, Pages M, Marty-Double C (1984) Congenital muscular dystrophy with cerebral white matter spongiosis. Brain Dev 6:491-495

9. Helbling-Leclerc A, Zhang X, Topaloglu H, Cruaud C, Tesson F, Weissenbach J, Tome FM, Schwartz K, Fardeau M, Tryggvason K et al (1995) Mutations in the laminin alpha 2-chain gene (LAMA2) cause merosin-deficient congenital muscular dystrophy. Nat Genet 11:216-218

10. Leegwater PA, Yuan BQ, van der Steen J, Mulders J, Konst AA, Boor PK, Mejaski-Bosnjak V, van der Maarel SM, Frants RR, Oudejans CB, Schutgens RB, Pronk JC, van der Knaap MS (2001) Mutations of MLC1 (KIAA0027), encoding a putative membrane protein, cause megalencephalic leukoencephalopathy with subcortical cysts. Am J Hum Genet 68:831-838

11. Leivo I, Engvall E (1988) Merosin, a protein specific for basement membranes of Schwann cells, striated muscle, and trophoblast, is expressed late in nerve and muscle development. Proc Natl Acad Sci USA 85:1544-1548

12. Lien CF, Hazai D, Yeung D, Tan J, Fuchtbauer EM, Jancsik V, Gorecki DC (2007) Expression of alpha-dystrobrevin in blood-tissue barriers: sub-cellular localisation and molecular characterisation in normal and dystrophic mice. Cell Tissue Res 327:67-82

13. Michele DE, Barresi R, Kanagawa M, Saito F, Cohn RD, Satz JS, Dollar J, Nishino I, Kelley RI, Somer H, Straub V, Mathews KD, Moore SA, Campbell KP (2002) Post-translational disruption of dystroglycan-ligand interactions in congenital muscular dystrophies. Nature 418:417-422

14. Muntoni F, Brockington M, Torelli S, Brown SC (2004) Defective glycosylation in congenital muscular dystrophies. Curr Opin Neurol 17:205-209

15. Nagelhus EA, Mathiisen TM, Ottersen OP (2004) Aquaporin-4 in the central nervous system: cellular and subcellular distribution and coexpression with KIR4.1. Neuroscience 129:905-913

16. Neely JD, Amiry-Moghaddam M, Ottersen OP, Froehner SC, Agre P, Adams ME (2001) Syntrophin-dependent expression and localization of Aquaporin-4 water channel protein. Proc Natl Acad Sci USA 98:14108-14113
17. Tachi N, Chiba S, Matsuo M, Matsumura K, Saito K (2001) Fukuyama muscular dystrophy associated with lack of C-terminal domain of dystrophin. Pediatr Neurol 24:373-378

18. Tachi N, Ohya K, Chiba S, Matsuo M, Patria SY, Matsumura K (1997) Deficiency of syntrophin, dystroglycan, and merosin in a female infant with a congenital muscular dystrophy phenotype lacking cysteine-rich and C-terminal domains of dystrophin. Neurology 49:579-583

19. Teijido O, Martinez A, Pusch M, Zorzano A, Soriano E, Del Rio JA, Palacin M, Estevez R (2004) Localization and functional analyses of the MLC1 protein involved in megalencephalic leukoencephalopathy with subcortical cysts. Hum Mol Genet 13:2581-2594

20. Tian M, Jacobson C, Gee SH, Campbell KP, Carbonetto S, Jucker M (1996) Dystroglycan in the cerebellum is a laminin alpha 2chain binding protein at the glial-vascular interface and is expressed in Purkinje cells. Eur J Neurosci 8:2739-2747

21. van der Knaap MS, Barth PG, Stroink H, van Nieuwenhuizen O, Arts WF, Hoogenraad F, Valk J (1995) Leukoencephalopathy with swelling and a discrepantly mild clinical course in eight children. Ann Neurol 37:324-334

22. van der Knaap MS, Barth PG, Vrensen GF, Valk J (1996) Histopathology of an infantile-onset spongiform leukoencephalopathy with a discrepantly mild clinical course. Acta Neuropathol (Berl) 92:206-212

23. van der Knaap MS, Smit LM, Barth PG, Catsman-Berrevoets CE, Brouwer OF, Begeer JH, de Coo IF, Valk J (1997) Magnetic resonance imaging in classification of congenital muscular dystrophies with brain abnormalities. Ann Neurol 42:50-59

24. van der Voorn JP, Pouwels PJ, Hart AA, Serrarens J, Willemsen MA, Kremer HP, Barkhof F, van der Knaap MS (2006) Childhood white matter disorders: quantitative MR imaging and spectroscopy. Radiology 241:510-517

25. van Kollenburg B, van Dijk J, Garbern J, Thomas AA, Scheper GC, Powers JM, van der Knaap MS (2006) Glia-specific activation of all pathways of the unfolded protein response in vanishing white matter disease. J Neuropathol Exp Neurol 65:707-715

26. Verbeek MM, Otte-Holler I, van den Born J, van den Heuvel LP, David G, Wesseling P, de Waal RM (1999) Agrin is a major heparan sulfate proteoglycan accumulating in Alzheimer's disease brain. Am J Pathol 155:2115-2125

27. Warth A, Kroger S, Wolburg H (2004) Redistribution of aquaporin-4 in human glioblastoma correlates with loss of agrin immunoreactivity from brain capillary basal laminae. Acta Neuropathol (Berl) 107:311-318

28. Warth A, Mittelbronn M, Wolburg H (2005) Redistribution of the water channel protein aquaporin-4 and the $\mathrm{K}+$ channel protein Kir4.1 differs in low- and high-grade human brain tumors. Acta Neuropathol (Berl) 109:418-426

29. Zaccaria ML, Di Tommaso F, Brancaccio A, Paggi P, Petrucci TC (2001) Dystroglycan distribution in adult mouse brain: a light and electron microscopy study. Neuroscience 104:311-324 